\title{
SpS1-From molecular clouds to massive stars
}

\author{
Maria R. Cunningham \\ School of Physics, University of New South Wales \\ Sydney, NSW, 2052, Australia \\ email: maria.cunningham@unsw.edu.au
}

\section{Introduction}

The life cycles of massive stars have a major impact on the evolution of galaxies, while in turn, position in galaxy has a major impact on the efficiency and type of star formation which occurs in a molecular cloud (see e.g. Luna et al. 2006). However, exactly how massive stars form, on what timescales, and how they shape their environments during this active and energetic phase is poorly understood.

\section{Stages of Massive Star Formation}

The processes leading from the formation of the molecular phase of the interstellar medium (ISM) to the formation of a massive star or cluster may be broken into a number of stages.

Formation of a giant molecular cloud: Giant molecular clouds (GMCs) are predominantly found in the spiral arms of the Galaxy, embedded in atomic hydrogen gas (HI), forming from $\mathrm{HI}$ in regions of enhanced density. The causes of the density enhancements are not known, but it is likely that turbulence, gravitational instability and converging flows of atomic gas all play a part (see e.g. Hennebelle et al. 2008). The origin of GMCs is important to the massive star formation story. Molecular clouds are turbulent over a wide range of environments and size scales (Heyer \& Brunt 2004), and turbulence likely plays a significant part in regulating star formation (see review by Mac Low \& Klessen 2004). The energy to drive turbulence must be constantly replenished, or the turbulent motions will quickly die out (see e.g. Stone, Ostriker, \& Gammie, 1998). While, the origin and size scales of the energy injection are unknown, there is now a consistent body of literature pointing to the likelihood that turbulence is predominantly driven at scales as large as or larger than the extent of the molecular component of the gas (Brunt, Heyer \& Mac Low 2009). Hence, the turbulent cascade may start in the atomic gas.

From giant molecular cloud to dense cores: There is a basic problem which needs to be explained by any model for massive star formation: In the Milky Way galaxy, only one star forms each year, on average, compared to the hundreds which should be forming if the gas in molecular clouds is simply collapsing under its own weight. Some physical process must act to limit the rate of star formation, and turbulence and magnetic fields have both been suggested as possibilities. Whether stars form: 1) quickly due to triggering by turbulent motions, with disruption by turbulent disturbances then limiting star formation efficiency, or 2) slowly due to gravitational collapse regulated by magnetic fields is debated (see e.g Elmegreen \& Scalo 2004; Mouschovias et al. 2006), although both phenomena are likely important (see e.g. Nakamura \& Li 2008).

In an effort to characterise turbulence observationally, we have mapped a $0.6 \times 1.2$ squaredegree region of the southern Galactic plane near G333, in molecular transitions near $3 \mathrm{~mm}$ that trace varying critical densities (e.g. ${ }^{13} \mathrm{CO}, \mathrm{CS}, \mathrm{N}_{2} \mathrm{H}^{+}$), using the Mopra radio telescope (Bains et al. 2006; Wong et al 2008; Lo et al 2009). We have used spatial power spectrum (Lazarian \& Pogosyan 2000) and delta variance (Bensch, Stutzki \& Ossenkopf 2001) analyses to follow the turbulent cascade through the molecular gas between the scales of $\sim 0.5 \mathrm{pc}$ to $20 \mathrm{pc}$. The results are reported elsewhere (Bains et al. 2009; Jones et al. in prep) but, in summary, all molecules show the same power spectrum slope, suggesting that: 1) turbulent energy is injected at scales larger than $\sim 20 \mathrm{pc}$ (consistent with Brunt et al. 2009), and 2) passes through the gas to the 
smallest scales examined $(\sim 0.5 \mathrm{pc})$ without dissipation, suggesting that the turbulent cascade continues to these smaller scales, affecting the formation of dense cores, and their fragmentation into individual protostars.

From dense cores to stars: The process by which a dense core evolves into a massive star or cluster is also debated. Carolan et al. (2009) find that massive star formation is most likely to occur by accretion occurring directly from the gas onto the protostar, producing a massive protostellar core, in what is often called the core accretion model. The discovery of an isolated massive star forming core in the G333 region (Lo et al. 2007) supports this. This is in contrast to the recent observations and simulations of Smith, Longmore \& Bonnell (2009), who find that the competitive accretion model seems more likely to apply. In this model, only small cores are able to form directly from the gas phase, as accretion is halted by radiation pressure once an object becomes too massive. Massive stars then form by the coalescence of these smaller cores.

\section{References}

Bains I. et al., 2006, MNRAS, 367, 1609

Bains I. et al., 2009, PASA, 26, 110

Bensch F., Stutzki J., \& Ossenkopf V., 2001, A\&A, 366, 636

Brunt, C. M., Heyer, M. H., \& Mac Low, M.-M. 2009, AछA, 504, 883

Carolan P. B. et al., 2009, MNRAS, 1353

Elmegreen, B. G. \& Scalo, J. 2004, ARAA, 42, 211

Hennebelle, P., Banerjee, R., Vázquez-Semadeni, E., Klessen, R. S., \& Audit, E. 2008, A\&\&A, 486, L43

Heyer, M. H. \& Brunt, C. M. 2004, ApJ, 615, L45

Lazarian A. \& Pogosyan D., 2000, ApJ, 537, 720

Lo N. et al., 2009, MNRAS, 395, 1021

Lo N., Cunningham M., Bains I., Burton M. G., \& Garay G., 2007, MNRAS, 381, L30

Luna A., Bronfman L., Carrasco L., \& May J., 2006, ApJ, 641, 938

Mac Low, M.-M., \& Klessen, R. S. 2004, Reviews of Modern Physics, 76, 125

Mouschovias, T. C., Tassis, K., \& Kunz, M. W. 2006, ApJ, 646, 1043

Nakamura, F. \& Li, Z.-Y. 2008, ApJ, 687, 354

Smith, R. J., Longmore, S., \& Bonnell, I. 2009, arXiv:0908.3910

Stone J. M., Ostriker E. C., \& Gammie C. F., 1998, ApJ, 508, L99

Wong T. et al., 2008, MNRAS, 386, 1069 